\title{
Análisis y evaluación de las competencias del Grado en Información y Documentación en la Universidad de Zaragoza
}

\section{Analysis and Evaluation of the Skills of the Degree in Information and Documentation at the University of Zaragoza}

María del Carmen Agustín Lacruz , José Antonio Salvador Oliván 2

' Departamento de Ciencias de la Documentación e Historia de la Ciencia, Universidad de Zaragoza, Spain. cagustin@unizar.es 2Departamento de Ciencias de la Documentación e Historia de la Ciencia, Universidad de Zaragoza, Spain. jaso@unizar.es

Resumen

Se estudian las competencias específicas descritas en las guías docentes publicadas del grado en Información y Documentación de la Universidad de Zaragoza. La metodología consiste en analizar las competencias recogidas en las guías docentes de todas las asignaturas mediante su inclusión en una base de datos y posterior procesado mediante el programa estadístico SPSS (V. 22.0). Entre los resultados destaca el estudio de 37 asignaturas. El número medio de competencias por asignatura es de 3,1. Las competencias con mayor presencia son Elaboración y difusión de la información, Conocimiento del entorno profesional de la información y documentación, Identificación, autentificación y evaluación de fuentes y recursos de información, y Organización y almacenamiento de la información, presentes en la tercera parte de las asignaturas del grado. En sentido negativo, las competencias que no están reflejadas en ninguna asignatura son Tecnologías de la información: Telecomunicaciones y Técnicas comerciales. En las asignaturas de Formación Básica, la competencias más frecuente es Elaboración y difusión de la información, que aparece en casi la mitad de las asignaturas de este tipo, seguida de las competencias Identificación autentificación y evaluación de recursos de información, Organización y almacenamiento de la información e Interacción con los productores, los usuarios y los clientes de información, que aparecen en casi la tercera parte de este tipo de asignaturas. En las asignaturas Obligatorias, la competencia más frecuente es Organización y almacenamiento de la información, que aparece en algo más de la tercera parte de las asignaturas. En las asignaturas Optativas, las competencias más frecuentes son Conocimiento del entorno profesional de la información y la documentación, Identificación, autentificación y evaluación de fuentes y recursos de información y Elaboración y difusión de la información.

Palabras clave

Competencias; Guías docentes; Grado en Información
Abstract

Specific skill described in the teaching guides of the Degree in Information and Documentation of the University of Zaragoza are studied. The methodology consists of analyzing the skills of all subjects by inclusion in a database and processed later by SPSS (v. 22.0). The results highlighted the study of 37 subjects. The average number of competitions per subject is 3.7. Skill with the largest presence are: Preparation and dissemination of information, Knowledge of the professional environment of Information and Documentation, Identification and evaluation of information sources and resources, and Organization and storage of Information. Skill without presence are: Information Technology: Telecommunications and Business skills. In the core subjects is the most common Preparation and dissemination of information. In the optional subjects, the most common skill are Knowledge of the professional environment of information and documentation, Identification, and evaluation of sources and resources of information and Preparation and dissemination of information.

Keywords 


\section{Introducción}

La reordenación de las titulaciones universitarias españolas promovida por la aplicación del Espacio Europeo de Educación Superior tuvo como objetivos principales facilitar la movilidad y la integración de los titulados en el mercado de trabajo europeo y adecuar el contenido de los estudios universitarios a las demandas sociales y laborales, mediante la adopción de un marco común de reconocimiento para que las titulaciones y las cualificaciones profesionales obtenidas sean comprensibles y comparables entre sí.

Una de las ideas motrices iniciales (Declaración de la Sorbona, 1998) fue impulsar también -junto a la moneda, la economía y las instituciones políticas comunes- una Sociedad europea del Conocimiento. El propósito era extender la educación superior a capas más amplias de la sociedad para incrementar el empleo ligado a la Economía del Conocimiento (Krüger, 2006) y fomentar la competitividad internacional de los sistemas educativos europeos, convirtiendo al viejo continente en foco de atracción para unos estudiantes, profesores y empleadores, obligados a desenvolverse en entornos cada vez más globalizados.

Para alcanzar estos objetivos era necesario reformular los planes de estudio, promover nuevos planteamientos didácticos para superar el mero dominio cognitivo de las disciplinas y propiciar la adquisición de competencias y aptitudes.

El nuevo modelo europeo de titulaciones sitúa al alumno en el centro del proceso educativo y pone en valor una nueva cultura de la calidad en las prácticas docentes del profesorado, tanto en lo que respecta a la descripción de competencias dentro de los diseños curriculares de cada titulación, como al establecimiento de los objetivos de enseñanza y los resultados de aprendizaje de asignaturas, como a la selección de contenidos y de estilos docentes.

En paralelo, los ejes de este nuevo paradigma de aprendizaje son la orientación hacia la empleabilidad en entornos socio-económicos globales y la consecución de competencias profesionales, entendidas en un sentido amplio como el conjunto de conocimientos, habilidades y actitudes que necesita una persona para ocupar adecuadamente un puesto de trabajo (Mora, 2011; Alonso, Fernández Rodríguez \& Nyssen, 2009, pp. 32-33).

En esencia, el proceso de Bolonia ha supuesto la transformación más importante ocurrida en la educación superior desde que las universidades europeas se adaptaron a la era industrial. Y el propósito es similar al ocurrido hace dos siglos: transmitir a los graduados las competencias que la nueva sociedad necesita (Mora, 2011, p. 29) para generar valor añadido en el sistema productivo 
y mejorar su competitividad (Declaración de Amberes, 2015). El escenario de los cambios se ha extendido también otras regiones geopolíticas, como los países latinoamericanos (Cortés Montalvo, 2012, p. 47).

El cambio de paradigma ha modificado la forma de pensar la estructura de las titulaciones y también la terminología utilizada: los antiguos planes de estudios se publicaban en los Boletines oficiales, a través de Órdenes Ministeriales o Decretos y recogían las relaciones de asignaturas, los descriptores con el contenido de las mismas y las adscripciones a las áreas de conocimiento con capacidad para impartirlas. En la actualidad la estructura curricular es más flexible y las titulaciones se organizan en torno a conceptos como competencia, materia, módulo, asignatura y guía docente (Agustín, Gómez \& Salvador, 2013, p. 38).

En este nuevo entorno educativo, las guías docentes de las asignaturas constituyen una valiosa herramienta didáctica que facilita el aprendizaje autónomo de los alumnos y el trabajo por competencias, definiendo la planificación de las clases y la "coreografía didáctica" de todo el proceso docente-discente. Contienen el programa desarrollado de cada asignatura, de manera que guían el aprendizaje y reconstruyen el itinerario que debe recorrer el alumno para superar la disciplina, describiéndolo desde su perspectiva. Incluyen datos descriptivos básicos sobre la materia y el profesorado que la imparte, el centro, el campus, la universidad, etc. También la contextualizan dentro de la titulación, el ciclo y el curso. Recogen el proyecto formativo de la materia, incluyendo los temas, las actividades a realizar y las orientaciones metodológicas generales y específicas. Finalmente, también establecen la bibliografía básica y el sistema, los criterios y el tipo de pruebas de evaluación. Este conjunto de información publicada, y con frecuencia difundida a través de la web, facilita la visibilidad de los programas de estudios y por tanto su conocimiento y comparación por parte de los alumnos interesados en cursarlas. Analizadas en su conjunto, permiten evidenciar que dentro del proyecto curricular de una titulación no existen solapamientos ni lagunas en el itinerario formativo que se ha diseñado (Agustín, 2008, p. 44).

Junto con el Proyecto de titulación, las guías docentes son el segundo gran instrumento de planificación y coordinación de las titulaciones. Las guías concretan y definen con precisión los resultados de aprendizaje, los sistemas de evaluación y los programas de actividades (Paricio Royo, 2010, p. 5). Constituyen una buena plataforma de observación y un excelente objeto de estudio para observar en ellas el alineamiento de las asignaturas respecto de las competencias que definen cada titulación. En la Universidad de Zaragoza, las guías docentes forman parte del sistema de calidad de las titulaciones y su redacción, aprobación y revisión está regulado por un procedimiento específico Q316 - dentro del Reglamento de la organización y gestión de la calidad de los estudios de grado y máster.

Por lo que respecta al Grado de Información y Documentación, ha aprovechado la adaptación al 
proceso de Bolonia, para cambiar su denominación inicial -Biblioteconomía y Documentaciónᄀ, escogiendo un nombre más inclusivo y genérico, así como para articular planes de estudios más integradores, con una orientación muy profesionalizante y para incorporar las tecnologías de forma transversal a la mayoría de las asignaturas (Abadal, 2013, pp. 216 y 217).

\section{Objetivos}

El propósito de este trabajo es revisar y analizar las competencias específicas descritas en las guías docentes publicadas de las asignaturas del grado en Información y Documentación impartido en la Universidad de Zaragoza -que se implantó en septiembre de 2008 y obtuvo la renovación de la acreditación en mayo de 2014-, para conseguir los siguientes objetivos específicos:

a) Comparar el grado de implantación de las competencias específicas el grado en Información y Documentación respecto de las señaladas en el Libro Blanco Título de Grado en Información y Documentación (ANECA, 2004).

b) Identificar las competencias no desarrolladas o desarrolladas de forma deficiente en las diversas asignaturas, para detectar posibles lagunas en la oferta formativa.

c) Evaluar si las competencias indicadas en las guías docentes de las asignaturas impartidas en el grado en la Universidad de Zaragoza se ajustan a los perfiles de trabajo de los profesionales de la información y la documentación.

\section{Material y métodos}

El desarrollo del trabajo se ha efectuado en las siguientes fases:

a) En primer lugar, se seleccionaron las guías docentes de las asignaturas de formación básica, obligatorias y optativas que se han impartido desde la implantación del grado en Información y Documentación en la Universidad de Zaragoza (curso 2008-2009).

Se han excluido las asignaturas Prácticum y Trabajo de Fin de Grado, las asignaturas optativas de idiomas (inglés, francés, alemán, italiano, árabe y catalán) y todas aquellas asignaturas optativas que, aunque forman parte del plan de estudios, no se han activado ni impartido nunca.

b) Todas las competencias específicas han sido redactadas por el profesorado de las asignaturas y aprobadas por los Consejos de Departamento responsables y por la Comisión de Garantía de Calidad del Grado. Las competencias están normalizadas, son las que figuran en la Memoria de 
Verificación del Grado y se ajustan a la clasificación del Libro Blanco del Grado en Información y Documentación (ANECA, 2004, pp. 155-178), salvo la competencia E24-Otros conocimientos aplicados a la información y documentación que no está presente en la Memoria del Grado.

Las competencias, y su significado, son las siguientes:

E01. Interacción con los productores, los usuarios y los clientes de la información. Analizar e interpretar las prácticas, las demandas, las necesidades y las expectativas de los productores, los usuarios y los clientes, actuales y potenciales, y desarrollar su cultura de la información ayudándoles a hacer el mejor uso de los recursos disponibles.

E02. Conocimiento del entorno profesional de la información y la documentación.

Orientarse en el entorno profesional nacional e internacional de la información y documentación, así como en su medio político, económico e institucional.

E03. Conocimiento del marco jurídico y administrativo nacional e internacional de la gestión de la información.

Aplicar las disposiciones y los procedimientos legales y reglamentarios tanto de ámbito nacional como internacional relativos a la actividad de información y documentación.

E04. Identificación, autentificación y evaluación de fuentes y recursos de información.

Identificar, evaluar y validar informaciones, documentos y sus fuentes, tanto internos como externos.

E05. Gestión de colecciones y fondos.

Elaborar y aplicar criterios de reunión, selección, adquisición y eliminación de documentos que permitan constituir y organizar colecciones de documentos de toda naturaleza o fondos de archivos, conservarlos haciéndolos accesibles, desarrollarlos teniéndolos al día y expurgarlos de elementos que se han convertido en inútiles, siguiendo la evolución de las necesidades de los usuarios.

E06. Preservación, conservación y tratamiento físico de documentos.

Definir y aplicar métodos y técnicas para ordenar, proteger, conservar, preservar y restaurar soportes documentales de cualquier naturaleza.

E07. Análisis y representación de la información.

Identificar y representar en el lenguaje documental adoptado o en otro sistema simbólico el contenido semántico de un documento o de una colección de documentos o de un fondo de archivo. 
E08. Organización y almacenamiento de la información.

Organizar y estructurar los dataos relativos a la descripción de documentos y colecciones de documentos en cualquier soporte; crear y explotar las herramientas de acceso a los datos, documentos o referencias.

E09. Búsqueda y recuperación de información.

Buscar y recuperar la información por métodos que permitan dar respuesta a las expectativas de los demandantes en condiciones óptimas de coste y tiempo.

E10. Elaboración y difusión de la información.

Hacer disponibles y explotables las informaciones tratadas y facilitar el uso mediante el suministro de productos y servicios documentales.

E17. Tecnologías de la información: informática.

Utilizar y poner en práctica métodos, técnicas y herramientas informáticas (hardware o software) para la implantación, desarrollo y explotación de sistemas de información.

E12. Tecnologías de la información: telecomunicaciones.

Utilizar y poner en práctica métodos, técnicas y herramientas informáticas (hardware o software) para la implantación, desarrollo y explotación de sistemas de telecomunicación.

E73. Técnicas de producción y edición.

Producir o reproducir documentos en cualquier soporte y formato con vistas a su difusión.

E14. Técnicas de gestión administrativa.

Garantizar el mantenimiento de la administración general, la gestión administrativa y el apoyo logístico de la actividad de un organismo.

E15. Técnicas de marketing.

Analizar y situar la actividad en un contexto estratégico y de competencia; promover dicha actividad elaborando y poniendo a punto las herramientas de trabajo apropiadas para la captación del mercado.

E16. Técnicas comerciales.

Establecer y mantener relaciones con clientes o socios con el fin de vender productos y servicios.

E17. Técnicas de adquisición.

Adquisición de los productos, documentos o prestaciones, en función de normas vigentes para su gestión y de una política de adquisiciones establecida. 
E18. Técnicas de gestión micro económica.

Controlar y optimizar de forma permanente los recursos del organismo y su utilización.

E19. Técnicas de instalación, acondicionamiento y equipamiento.

Técnicas de instalación, acondicionamiento y equipamiento.

E20. Técnicas de planificación y gestión de proyectos.

Prever, organizar, gestionar y llevar a buen término un proyecto técnico integrando las limitaciones del entorno: humanas, económicas, de calendario, reglamentarias, etc.

E21. Técnicas de diagnóstico y evaluación.

Identificar los puntos fuertes y débiles de una organización, de un producto o de un servicio, establecer y utilizar indicadores, elaborar soluciones para mejorar la calidad.

E22. Técnicas de gestión de recursos humanos.

Asegurar la integración, la eficacia y el bienestar del personal de una unidad de trabajo, aplicando la legislación y la reglamentación en vigor, respetando los objetivos de la empresa, favoreciendo el desarrollo personal y profesional de los individuos.

E23. Técnicas de formación.

Concebir y ejecutar una acción o un plan de formación inicial o continua.

Se introdujeron en una base de datos todas las competencias específicas presentes en las asignaturas seleccionadas y las competencias transversales recogidas en el Libro Blanco Título de Grado en Información y Documentación (ANECA, 2004, p. 148).

c) Finalmente se procesó la información con el programa estadístico SPSS (v. 22.0).

\section{Resultados}

\subsection{Plan de estudios del grado en Información y Documentación en la universidad de Zaragoza}

El número total de créditos es de 240, distribuidos de manera equitativa en los 4 cursos. En primer y segundo cursos, la mitad de los créditos se corresponde con asignaturas de formación básica y la otra mitad con asignaturas obligatorias.

En tercer curso, 42 créditos son de asignaturas obligatorias y 18 de asignaturas optativas; en cuarto curso, la mayoría de los créditos que debe cursar un alumno son de asignaturas optativas, y tan solo 18 créditos son de asignaturas obligatorias (12 del Prácticum y 6 del Trabajo de fin de grado). 


\begin{tabular}{|c|c|c|c|}
\hline Cursos/Tipo de asignaturas & Formación Básica & Obligatorias & \multirow{2}{*}{ Optativas } \\
\cline { 1 - 3 } Primer curso & $5(30$ créditos $)$ & $4(30$ créditos $)$ & \\
\cline { 1 - 3 } Segundo curso & $5(30$ créditos $)$ & $4(30$ créditos $)$ & \\
\cline { 1 - 3 } Tercer curso & & $6(42$ créditos $)$ & \multirow{2}{*}{$13(78$ créditos $)$} \\
\cline { 1 - 3 } Cuarto curso & & $2(18$ créditos $)$ & \\
\hline
\end{tabular}

Tabla 1. Distribución del número y tipo de asignaturas y créditos por cursos

El número total de asignaturas en las que se han analizado las competencias es de 37 . El número medio de competencias presentes en las asignaturas es de 3,1, teniendo la mayoría de ellas (el 83,7\%) entre 2 y 4 competencias (Gráfico 1).

Las asignaturas con mayor número de competencias son:

- $\quad$ El libro antiguo en los sistemas de información, optativa de 6 créditos, con 9 competencias. - $\quad$ Organización y gestión de archivos, obligatoria de $1^{\circ}$ curso con 9 créditos, Conservación preventiva en archivos y bibliotecas, obligatoria de $3^{\circ}$ curso y Paleografía general, optativa de 6 créditos. Todas ellas con 5 competencias.

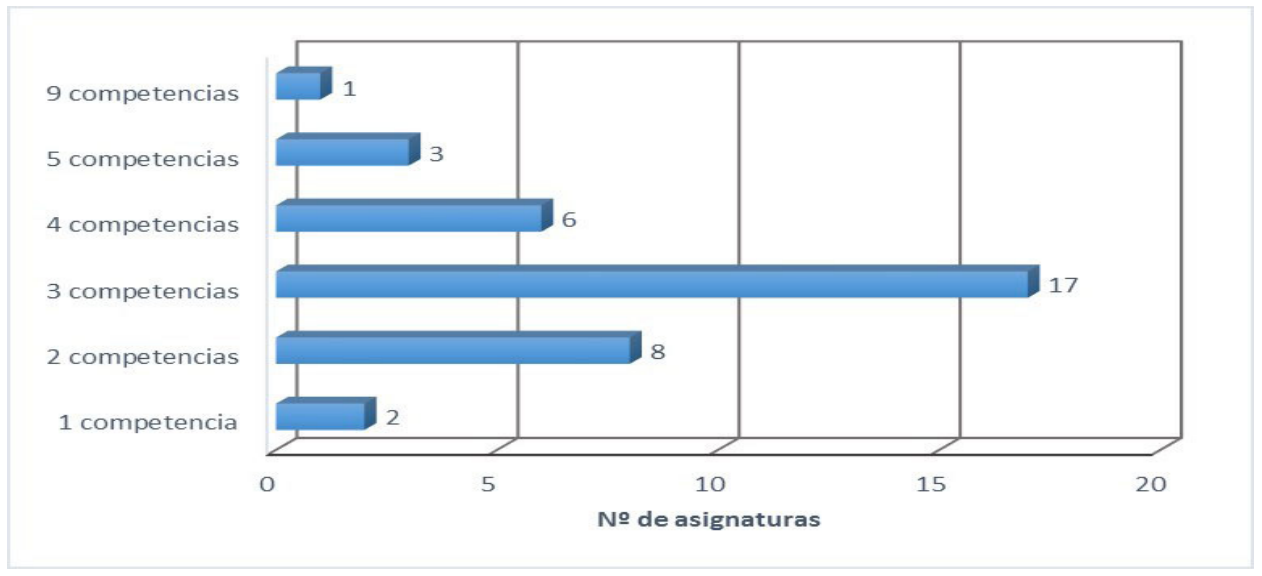

Gráfico 1. Distribución del número de competencias en asignaturas

En 18 asignaturas (48,6\%) no se indica ninguna competencia transversal, mientras que en 19 asignaturas sí aparecen de manera explícita este tipo de competencias, siendo la media de 3,4 competencias transversales (entre 1 y 7 competencias). Las asignaturas de Formación básica son las que más presencia de competencias transversales tienen (8 de 10), en las obligatorias aparecen en 9 de 14, y en las optativas solo aparecen en 2 asignaturas de 13. 


\subsection{Análisis de las competencias en las asignaturas}

En la tabla 2 se muestran las competencias con mayor presencia en las asignaturas. Destacan las competencias Elaboración y difusión de la información, Conocimiento del entorno profesional de la información y documentación, Identificación, autentificación y evaluación de fuentes y recursos de información, y Organización y almacenamiento de la información, presentes en la tercera parte de las asignaturas del grado.

En sentido negativo, dos competencias no están reflejadas en ninguna asignatura: Tecnologías de la información: Telecomunicaciones y Técnicas comerciales. Sorprende que la primera de ellas no esté representada en ninguna asignatura ya que es específica de la materia troncal Tecnologías de la información y edición digital, con contenidos muy relevantes en la actualidad y una propuesta de 24 créditos en la titulación.

La competencia Técnicas comerciales no está representada en ninguna asignatura, y junto a las relacionadas con las de marketing, adquisición, gestión y formación forman parte de una única materia del Libro Blanco, Planificación, organización y evaluación de unidades de información, y todas hacen referencia a la venta, adquisición y control de productos y servicios.

En las asignaturas de Formación Básica, la competencias más frecuente es Elaboración y difusión de la información, que aparece casi la mitad de las asignaturas de este tipo (tres de segundo curso y una de primero), seguida de las competencias Identificación autentificación y evaluación de recursos de información, Organización y almacenamiento de la información e Interacción con los productores, los usuarios y los clientes de información, que aparecen en casi la tercera parte de este tipo de asignaturas.

En las asignaturas Obligatorias, la competencia más frecuente es Organización y almacenamiento de la información, que aparece en algo más de la tercera parte de las asignaturas. En este tipo de asignaturas están representadas 16 competencias, faltando, además de las dos que no aparecen en ninguna asignatura, las de Técnicas de gestión administrativa, Técnicas de marketing, Técnicas de gestión microeconómica, Técnicas de gestión de recursos humanos y Técnicas de formación, competencias que sí aparecen en asignaturas de formación básica.

En las asignaturas Optativas, las competencias más frecuentes son Conocimiento del entorno profesional de la información y la documentación, Identificación, autentificación y evaluación de fuentes y recursos de información y Elaboración y difusión de la información, presentes en la mitad de este tipo de asignaturas. Destaca que 8 competencias no aparezcan en ninguna asignatura optativa, y la poca presencia de aquellas relacionadas con las Tecnologías de la información y Técnicas de producción 
y edición, y que están directamente relacionadas con uno de los perfiles de salida de la titulación, concretamente con el gestor de contenidos en el entorno web.

\begin{tabular}{|l|c|}
\hline & $N^{0}$ de asignaturas \\
\hline E10. Elaboración y difusión de la información & 13 \\
\hline E02. Conocimiento del entorno profesional de la información y la documentación & 12 \\
\hline E04. Identificación, autentificación y evaluación de fuentes y recursos de información & 12 \\
\hline E08. Organización y almacenamiento de la información & 12 \\
\hline E07. Análisis y representación de la información & 10 \\
\hline E05. Gestión de colecciones y fondos & 8 \\
\hline E21. Técnicas de diagnóstico y evaluación & 8 \\
\hline E01. Interacción con los productores, los usuarios y los clientes de la información & 7 \\
\hline E03. Conocimiento del marco jurídico y administrativo nacional e internacional de la gestión de \\
la información & 6 \\
\hline E09. Búsqueda y recuperación de información & 5 \\
\hline E06. Preservación, conservación y tratamiento físico de documentos & 5 \\
\hline E20. Técnicas de planificación y gestión de proyectos & 3 \\
\hline E11. Tecnologías de la información: informática & 3 \\
\hline E13. Técnicas de producción y edición & 1 \\
\hline E14. Técnicas de gestión administrativa & 1 \\
\hline E15. Técnicas de márketing & 1 \\
\hline E17. Técnicas de adquisición & 1 \\
\hline E18. Técnicas de gestión microeconómica & 1 \\
\hline E19. Técnicas de instalación, acondicionamiento y equipamiento & 1 \\
\hline E22. Técnicas de gestión de recursos humanos & 1 \\
\hline E23. Técnicas de formación & 0 \\
\hline E12. Tecnologías de la información: telecomunicaciones & 0 \\
\hline E16. Técnicas comerciales & \\
\hline
\end{tabular}

Tabla 2. Presencia de las competencias en las asignaturas del grado en Información y Documentación

\begin{tabular}{|l|c|}
\hline & $N^{0}$ de asignaturas \\
\hline E10. Elaboración y difusión de la información & 4 \\
\hline E01. Interacción con los productores, los usuarios y los clientes de la información & 3 \\
\hline E04. Identificación, autentificación y evaluación de fuentes y recursos de información & 3 \\
\hline E08. Organización y almacenamiento de la información & 3 \\
\hline E02. Conocimiento del entorno profesional de la información y la documentación & 2 \\
\hline E07. Análisis y representación de la información & 2 \\
\hline E21. Técnicas de diagnóstico y evaluación & 2 \\
\hline $\begin{array}{l}\text { E03. Conocimiento del marco jurídico y administrativo nacional e internacional de la gestión de } \\
\text { la información }\end{array}$ & 1 \\
\hline E09. Búsqueda y recuperación de información & 1 \\
\hline E11. Tecnologías de la información: informática & 1 \\
\hline E13. Técnicas de producción y edición & 1 \\
\hline E14. Técnicas de gestión administrativa & 1 \\
\hline E15. Técnicas de márketing & 1 \\
\hline E18. Técnicas de gestión microeconómica & 1 \\
\hline E22. Técnicas de gestión de recursos humanos & 2 \\
\hline
\end{tabular}

Tabla 3. Presencia de las competencias en las asignaturas de Formación Básica del grado en Información y Documentación 


\begin{tabular}{|l|c|}
\hline & $N^{0}$ de asignaturas \\
\hline E08. Organización y almacenamiento de la información & 5 \\
\hline E02. Conocimiento del entorno profesional de la información y la documentación & 4 \\
\hline E07. Análisis y representación de la información & 4 \\
\hline E21. Técnicas de diagnóstico y evaluación & 4 \\
\hline E05. Gestión de colecciones y fondos & 4 \\
\hline E10. Elaboración y difusión de la información & 3 \\
\hline E04. Identificación, autentificación y evaluación de fuentes y recursos de información & 3 \\
\hline E01. Interacción con los productores, los usuarios y los clientes de la información & 3 \\
\hline E09. Búsqueda y recuperación de información & 2 \\
\hline E20. Técnicas de planificación y gestión de proyectos & 2 \\
\hline $\begin{array}{l}\text { E03. Conocimiento del marco jurídico y administrativo nacional e internacional de la gestión de } \\
\text { la información }\end{array}$ & 1 \\
\hline E11. Tecnologías de la información: informática & 1 \\
\hline E13. Técnicas de producción y edición & 1 \\
\hline E06. Preservación, conservación y tratamiento físico de documentos & 1 \\
\hline E17. Técnicas de adquisición & 1 \\
\hline E19. Técnicas de instalación, acondicionamiento y equipamiento & 1 \\
\hline
\end{tabular}

\begin{tabular}{|l|c|}
\hline & $N^{0}$ de asignaturas \\
\hline E02. Conocimiento del entorno profesional de la información y la documentación & 6 \\
\hline E04. Identificación, autentificación y evaluación de fuentes y recursos de información & 6 \\
\hline E10. Elaboración y difusión de la información & 6 \\
\hline E05. Gestión de colecciones y fondos & 4 \\
\hline E06. Preservación, conservación y tratamiento físico de documentos & 4 \\
\hline E07. Análisis y representación de la información & 4 \\
\hline E08. Organización y almacenamiento de la información & 4 \\
\hline $\begin{array}{l}\text { E03. Conocimiento del marco jurídico y administrativo nacional e internacional de la gestión de } \\
\text { la información }\end{array}$ & 3 \\
\hline E09. Búsqueda y recuperación de información & 3 \\
\hline E20. Técnicas de planificación y gestión de proyectos & 3 \\
\hline E21. Técnicas de diagnóstico y evaluación & 1 \\
\hline E01. Interacción con los productores, los usuarios y los clientes de la información & 1 \\
\hline E11. Tecnologías de la información: informática & 1 \\
\hline E13. Técnicas de producción y edición & 1 \\
\hline E23. Técnicas de formación & \\
\hline
\end{tabular}

Tabla 5. Presencia de las competencias en las asignaturas Optativas del grado en Información y Documentación 


\subsection{Análisis de las competencias por cursos}

En el análisis por cursos (Gráfico 2), destaca que solo 8 competencias (poco más del 1/3 del total) aparecen en todos los cursos. Las competencias E01, E04, E05, E07, E08, E10 y E21 están representadas en materias troncales que en la propuesta del Libro Blanco están cubiertas, al menos, por 48 créditos obligatorios, lo que puede explicar su presencia en los 4 cursos del grado. La competencia E02 aparece en la propuesta del Libro Blanco solo en 12 créditos obligatorios, y aunque se adquiere sobre todo en asignaturas optativas, al ser tan generalista muy probablemente esté sobrerrepresentada y sea un cajón de sastre de muchas asignaturas.

Sin embargo, la competencia E02 (Conocimiento del entorno profesional de la información y la documentación) solo está representada en la materia troncal de Documentos, unidades y sistemas de información, con una propuesta de 12 créditos, equivalentes a 2 asignaturas, y por tanto, resulta claramente insuficiente para que estas competencias puedan estar representadas en los 3 primeros cursos con asignaturas obligatorias, ya que sí hay optativas con estas competencias. Igualmente ocurre con la competencia E03 Conocimiento del marco jurídico y administrativo de la gestión de la información.

El número de competencias diferentes por cursos es el mismo en primero y en tercero (12), y unas pocas más en segundo y en cuarto (optativas), con 15 competencias en cada uno e ellos.

En cuarto curso, donde hay un mayor número de asignaturas (13 frente a 9 en primero y segundo, y 6 en tercer curso), se podría esperar que hubiera también un mayor número de competencias, sobre todo porque todas las asignaturas son optativas y deberían estar especializadas en aspectos concretos de la profesión y cubrir competencias muy específicas que no están representadas en asignaturas de cursos anteriores.

\subsection{Ajuste de las competencias al Libro Blanco}

En la siguiente tabla se compara el grado de adecuación de la presencia de competencias medido en número de créditos en las asignaturas del grado de la universidad de Zaragoza y la propuesta de contenidos comunes obligatorios realizada en el Libro Blanco. Teniendo en cuenta solo las asignaturas de formación básica y obligatorias (FB + Obl.), se observa que hay un grado de correspondencia muy alto en las competencias de Organización y almacenamiento de la información, Análisis y representación de la información, e Interacción con los productores, usuarios y clientes de la información.

La competencia Conocimiento del entorno profesional de la información y documentación está mucho más 


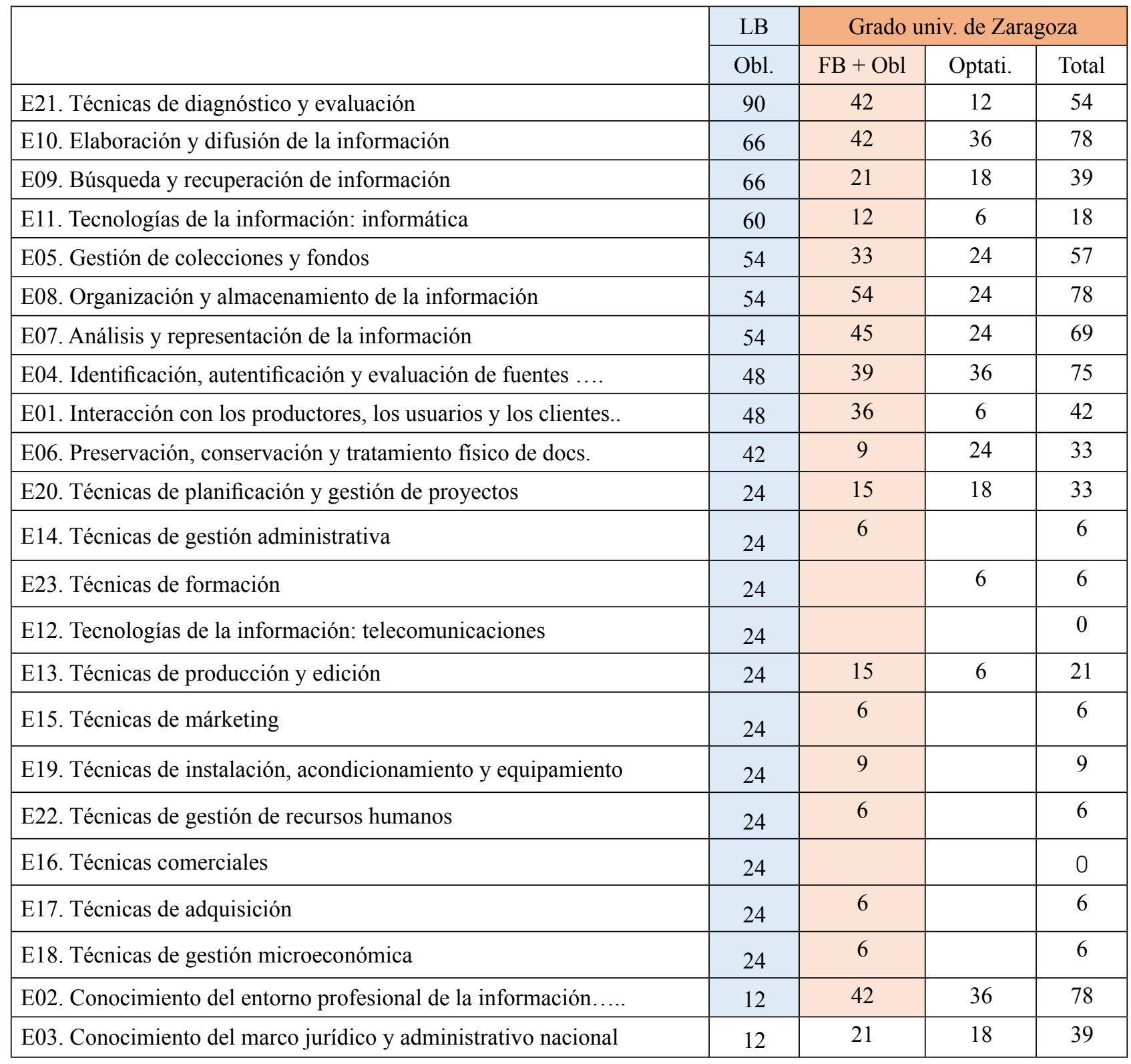

Tabla 6. Número de créditos dedicados a la adquisición de competencias según la propuesta de contenidos comunes obligatorios del Libro Blanco (LB) y el Grado en Información y Documentación de la universidad de Zaragoza

desarrollada que en el Libro Blanco, hasta en 7 asignaturas diferentes, y algo menos la competencia Conocimiento del marco jurídico y administrativo nacional. Que estas competencias estén mucho más desarrolladas en el grado puede estar indicando un solapamiento de contenidos en distintas asignaturas o que, en el primer caso, sea una competencia tan general y básica que es adoptada en muchas guías docentes de aquellas asignaturas en las que se introduce a la documentación.

Por otra parte, son muchas las competencias que están infrarrepresentadas, destacando sobre todo las de Técnicas de diagnóstico y evaluación, Búsqueda y recuperación de la información, Tecnologías de la información, y Preservación, conservación y tratamiento físico de documentos, que no llegan a la propuesta del Libro Blanco ni sumando los créditos de las asignaturas optativas en las que se imparten. Estas competencias se consideran de especial importancia en los distintos perfiles de los 
graduados en información y documentación por lo que se hace necesario averiguar las causas del bajo desarrollo de estas competencias, bien por falta de contenidos adecuados en asignaturas o por una deficiente identificación de competencias en las guías docentes.

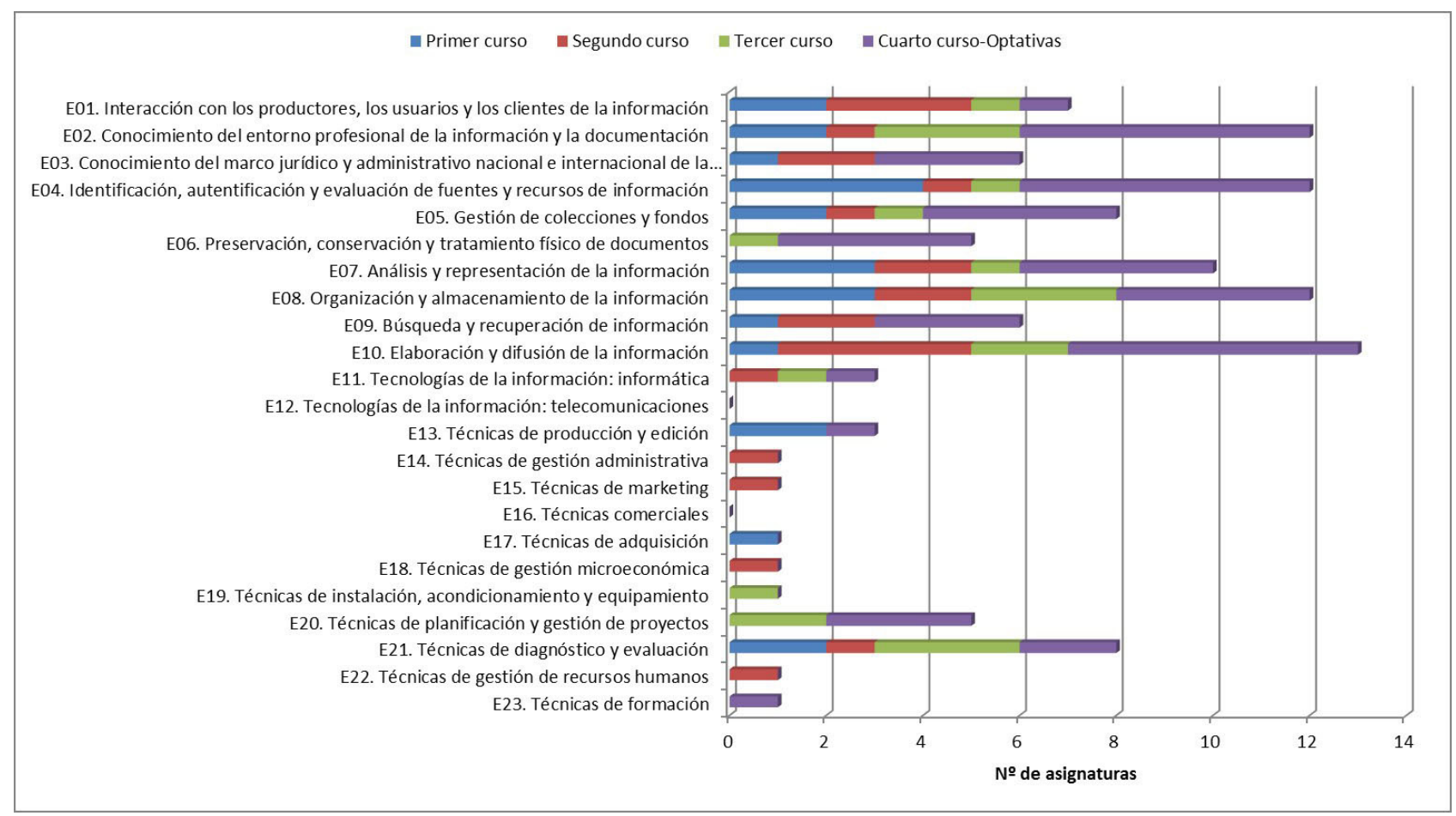

Gráfico 2. Distribución de las competencias por cursos

\section{Conclusiones}

Los resultados obtenidos del análisis de las competencias permiten, de manera global, mejorar la calidad del grado, y de manera específica, poner en marcha medidas correctoras que palien los déficits observados.

En general, las guías docentes del Grado en Información y Documentación de la Universidad de Zaragoza contienen información de gran interés y utilidad para los alumnos, lo más completa y exacta posible. Un apartado esencial es el que ofrece información sobre las competencias que adquiere el alumno en cada una de las asignaturas de la titulación, por ello es de capital importancia que las competencias estén descritas de manera adecuada y normalizada, y que estén todas las que son y sean todas las que estén.

En el grado en Información y Documentación de la Universidad de Zaragoza, se observan 2 competencias ausentes: Tecnologías de la información: Telecomunicaciones y Técnicas comerciales.

La primera de ellas es una competencia necesaria e importante en trabajos relacionados con perfiles profesionales actuales como la gestión de contenidos. Por ello es necesario revisar los programas 
de las guías docentes de asignaturas obligatorias que deberían cubrir esta competencia y hacerla explícita, pues dado el carácter instrumental y transversal de esta competencia, podría ser en realidad una omisión, más que una carencia real. En cualquier caso, es necesario revisar la incorporación de esta competencia en las guías docentes y si fuera necesario, activar asignaturas optativas como Sistemas, redes y consorcios de bibliotecas, o Técnicas informáticas de soporte para productos y servicios documental, que proporcionan una formación importante para el profesional de la Información y Documentación.

Sobre la competencia Técnicas comerciales es necesario reflexionar acerca de si realmente es necesaria en perfiles laborales de Información y Documentación y, si es así, incluirlas en contenidos de alguna asignatura obligatoria como puede ser la de Dirección de unidades de información o Planificación y evaluación de unidades de información, o bien activar la asignatura optativa donde puede tener cabida como es la de Gestión de recursos en unidades de información.

Las competencias recogidas en el Libro Blanco, a excepción de las dos competencias señaladas anteriormente, están implantadas y se desarrollan en las asignaturas del grado de la universidad de Zaragoza, si bien el grado de adecuación o representación es inadecuado. Es necesario llevar a cabo otro estudio complementario a este que permita averiguar las causas, que pueden deberse a una deficiente identificación de las competencias en las guías docentes (es decir, que se desarrollan competencias que no están señaladas en las guías) o bien a que faltan contenidos que permitan a los estudiantes adquirir de una manera más completa las competencias, lo que inevitablemente llevaría a modificar los programas de algunas asignaturas al tratarse de competencias desarrolladas en contenidos obligatorios.

Se considera necesario reforzar aquellas competencias con menos presencia activando asignaturas optativas e incluso reformando el plan de estudios fusionando las dos asignaturas de Instituciones españolas y europeas, de formación básica como Historia de las instituciones españolas hasta el siglo XX, sin competencias específicas en la titulación, y liberar créditos que podrían utilizarse para desarrollar otras asignaturas con competencias más necesarias.

La mayoría de las competencias estudiadas se adecuan a los perfiles de trabajo de los profesionales de la Información, en servicios y sistemas de información, archivos, bibliotecas y centros de documentación. No obstante, transcurrida más de una década desde la publicación del Libro Blanco de la titulación, resulta ineludible proceder a actualizar y adaptar sus directrices a los entornos profesionales actuales. 


\section{Referencias}

Abadal Falgueras, E. (2013). La Biblioteconomía y la Documentación en la universidad española de principios del siglo XXI. Nuovi Annali della Scouola Speciale per archivisti e bibliotecari, 27, 211-228.

Agustín Lacruz, M. ${ }^{a}$ C. (coord.) (2008). Diseño curricular y guías docentes ECTS: desde la Diplomatura de Biblioteconomía y Documentación hasta el Grado en Información y Documentación. Zaragoza: Prensas Universitarias de Zaragoza.

Agustín Lacruz, M. a C., Gómez Díaz, R. \& Salvador Oliván, J. A. (2013). Competencias para el análisis, la representación, la organización y la recuperación de la información en los Grados de Información y Documentación en España. En F. Ribeiro \& E. Cerveira (org.), Informaçâo e/ou conhecimento: as duas faces de Jano. Atas del I Congreso ISKO Espanha e Portugal - XI Congreso ISKO España (pp. 35-48). Porto: Cetac.Media.

Alonso, L. E.; Fernández Rodríguez, C. J. \& Nyssen, J. M. (2009). El debate sobre las competencias: una investigación cualitativa en torno a la educación superior y el mercado de trabajo en España. Madrid: ANECA. Recuperado el 9 de julio de 2015 de http://www.aneca.es/media/148145/publi_ competencias_090303.pdf.

ANECA (2004). Título de Grado en Información y Documentación. Madrid: Aneca. Recuperado el 22 de mayo de 2015 de http://www.aneca.es/media/150424/libroblanco_jun05_documentacion.pdf.

Cortés Montalvo, J. A. (2012). La competencia comunicativa en la formación universitaria. En C. Marta Lazo, M. ${ }^{a}$ C. Agustín Lacruz \& I. Ubieto Artur (coords.), Competencias interdisciplinares para la comunicación y la información en la sociedad digital (pp. 47-72). Madrid: Icono14.

EUROPEAN UNIVERSITY ASSOCIATION (2015). EUA Antwerp Declaration. A strategic agenda for universities: Developing strong institutions to advance the knowledge-based society. Recuperado el 10 de julio 2015 de http://www.eua.be/Libraries/Project_logos_images/Antwerp_Declaration_21_05_2015_ final.sflb.ashx.

Krüger, K. (2006). El concepto de 'Sociedad del Conocimiento'. BIBLIO 3W: Revista Bibliográfica de Geografía y Ciencias Sociales, 17(683). Recuperado el 1 de julio 2015 de http://www.ub.edu/geocrit/ b3w-683.htm. 
Mora, J. G. (2011). Formando en competencias: ¿un nuevo paradigma? Barcelona: Fundación CYD. Colección documentos CYD 15/2011. Recuperado el 1 de julio 2015 de http://www.fundacioncyd.org/ images/documentosCyd/CYD15.pdf.

Paricio Royo, J. (2010). Un modelo de guía docente desde los resultados de aprendizaje y su evaluación. Zaragoza: Instituto de Ciencias de la Educación. Documentos de referencia para la calidad docente. Recuperado el 6 de julio 2015 de http://www.unizar.es/ice/images/stories/calidad/Modelo\%20 gu\%C3\%ADa\%20docente.pdf. 